ISSN 1983-8484

Licenciado sob uma Licença Creative Commons

\title{
Sistema inteligência setorial: acesso e compartilhamento de informação e conhecimento para a obtenção de vantagem competitiva
}

\author{
Sector intelligence system: access and sharing of information \\ and knowledge to achieve competitive
}

Douglas Luis Três ${ }^{[a]}$, Marcondes da Silva Cândido ${ }^{[b]}$

[a] Mestre em Administração pela Universidade Federal de Santa Catarina (UFSC), Florianópolis, SC - Brasil, e-mail: douglas@sc.sebrae.com.br

[b] Doutorando em Engenharia e Gestão do Conhecimento pela Universidade Federal de Santa Catarina (UFSC), Florianópolis, SC Brasil, e-mail: marcondes@sc.sebrae.com.br

\section{Resumo}

Este estudo objetivou descrever o Projeto SIS SEBRAE/SC como ferramenta inovadora que proporciona o acesso e compartilhamento de informação e conhecimento entre as empresas associadas em Arranjos Produtivos Locais (APLS), bem como a obtenção de vantagem competitiva por meio da inteligência gerada. A estratégia do projeto se apoia em um Portal no qual as empresas têm a oportunidade de compartilhar informações, tratadas e analisadas por uma equipe de especialistas que as converte em conhecimento. A intenção é disponibilizar um instrumento de apoio à tomada de decisão e proporcionar a colaboração interorganizacional. Essa colaboração acontece, principalmente, pelo uso comum dos produtos de inteligência e pela utilização dos demais recursos oferecidos no portal. 0 método de pesquisa é um estudo de caso, e para tanto foi analisado o framework do projeto, bem como foram descritos os produtos de inteligência gerados pelo sistema. Pode-se dizer que a IC setorial visa tornar todo o setor mais competitivo. A pesquisa mostra que é possível estruturar com sucesso um modelo de inteligência competitiva que aborde um setor ou segmento da economia, e também é observado que a criação de canais de comunicação com os usuários (modelo de relatório Ad-hoc) é fundamental para customizar a informação e garantir a satisfação dos usuários.

Palavras-chave: Inteligência competitiva setorial. Sistema de inteligência setorial. Arranjo produtivo local. Colaboração interorganizacional.

\footnotetext{
Abstract

This study aimed to describe the Project SIS SEBRAE/SC as an innovative instrument that provides access and sharing of information and knowledge among member companies in Arranjos Produtivos Locais, (Cluster) - APL, as well as to obtain a competitive advantage through intelligence generated. The project's strategy is supported on a website, where companies have the opportunity to share information analyzed by a team of experts who converts to knowledge. The intention is to offer a tool to support decision making and provide inter-collaboration. This collaboration
} 
takes place mainly by the common use of intelligence products and the use of other features offered on the website. The research method is a case study to this purpose, we analyzed the framework of the project, and were described the intelligence products generated by the system. The research shows that the Intelligence Competitive aims at making the whole sector more competitive. It's shows that it is possible to structure a successful model of competitive intelligence that addresses a sector or segment of the economy, is also observed that the creation of channels of communication with users (Model Ad-hoc report) is critical to customize the information and guarantee consumer satisfaction.

Keywords: Industry competitive intelligence. Industry intelligence system. Cluster. Inter-collaboration.

\section{Introdução}

A informação permeia todas as atividades organizacionais e a cada dia vem ganhando maior importância nas organizações. Os gestores percebem que gerenciar o fluxo informacional e monitorar o ambiente externo é fundamental para o bom desempenho gerencial. Nessa perspectiva, o processo de inteligência competitiva (IC) vem ganhando força na busca pela diminuição das incertezas, monitoramento da concorrência, identificação das oportunidades e ameaças, bem como para auxiliar a tomada de decisão. Ao utilizar o conhecimento gerado a partir do processo de IC, a organização pode se posicionar à frente de seus concorrentes e obter vantagem competitiva.

As micro e pequenas empresas (MPE) têm papel significativo na economia do país, entretanto, a cada dia podem ser fortemente atingidas pela concorrência acirrada do mercado, devido a sua estrutura menor e muitas vezes pouco consolidada. Machado e Abreu (2009) afirmam que com a aceleração da globalização, a competição deixar de ser com o vizinho ao lado e ganha uma amplitude maior, pode ser com outra região do planeta que obteve maior produtividade, com produtos e serviços a custo menor.

Para auxiliar as MPE, o Serviço Brasileiro de Apoio às Micro e Pequenas Empresas (SEBRAE) tem buscado alternativas para melhorar o desempenho e posicionamento dessas empresas no mercado. Uma delas é a criação de sistemas de IC setorial junto aos Arranjos Produtivos Locais (APL). Neste artigo será apresentado o Sistema de Inteligência Setorial (SIS) voltado para os APLs de vestuário, calçados femininos, móveis de madeira e apicultura, ligados ao SEBRAE de Santa Catarina (SC).

O objetivo geral desta pesquisa é descrever o SIS SEBRAE/SC como ferramenta inovadora que proporciona o acesso e compartilhamento de informação e conhecimento entre as empresas associadas aos APLs, bem como a obtenção de vantagem competitiva por meio da inteligência gerada.

Dispor de informação relevante e no momento certo significa, para a organização, estar munida de subsídios que proporcionarão a comunicação e maior segurança para chegar a um objetivo comum. Torna-se possível aos tomadores de decisão economizar tempo e fazer escolhas a partir de informações estruturadas e com valor agregado.

As empresas vinculadas ao SIS têm a possibilidade de obter informação atual e de qualidade conforme suas necessidades, bem como tomar conhecimento do ambiente onde estão inseridas para estarem preparadas para as diversas situações. Essas organizações podem solicitar a informação de que precisam, ter exclusividade sobre ela e posteriormente compartilhar com o APL, buscando o desenvolvimento conjunto.

Seguindo esta introdução, apresenta-se o referencial teórico sobre a temática IC setorial, posteriormente o Portal SIS é apresentado e contextualizado como ferramenta inovadora e que proporciona o compartilhamento de informação e conhecimento. Por fim, são apresentadas as considerações finais.

\section{Inteligência competitiva setorial}

Frente ao novo cenário econômico e à vasta disponibilidade de informação, as organizações passam a buscar meios de melhorar o tratamento e seleção das informações que podem auxiliar os gestores na tomada de decisão e, consequentemente, proporcionar o alcance da vantagem competitiva.

Porter e Millar (1999) defendem a ideia de que o impacto da tecnologia é tão difuso que os executivos se defrontaram com o problema do excesso de informação, o que leva a novos usos para a tecnologia 
da informação e comunicação (TIC), em termos de armazenamento e análise das inúmeras informações disponíveis. Eles ainda argumentam que a revolução da informação afeta a competição de três maneiras: muda a estrutura setorial e, assim, altera as regras da competição; gera vantagem competitiva ao proporcionar às empresas novos modos de superar o desempenho dos rivais; dissemina negócios inteiramente novos, em geral a partir das atuais operações da empresa.

Um processo que se mostra cada vez mais eficiente para subsidiar essa necessidade das organizações é a IC. Inúmeros conceitos são apresentados na literatura para descrever esse processo e aqui serão apresentados alguns para fins deste estudo.

IC é um processo sistemático que transforma bits aleatórios e pedaços de dados em conhecimento estratégico. É informação sobre as forças dentro do mercado, sobre produtos específicos e tecnologia, é também informação externa, por exemplo, econômica, regulatória, política e demográfica, as quais têm um impacto sobre o mercado (TYSON, 1998, tradução nossa).

Kahaner (1996), outro autor expressivo da área, afirma que "IC é um programa sistemático de coleta e análise de informação sobre as atividades, tendências do mercado e sobre os competidores para alcançar os objetivos da organização" (tradução nossa).

A IC também é entendida como uma nova síntese teórica no tratamento da informação, uma metodologia que permite o monitoramento informacional da ambiência e, quando sistematizado e analisado, a tomada de decisão (TARAPANOFF, 2001).

No conceito apresentado por Gomes e Braga (2004), as autoras afirmam que além de um processo sistemático, IC também é um processo ético de identificação, coleta, tratamento, análise e disseminação da informação estratégica para a organização, viabilizando seu uso no processo decisório. Outra questão importante ressaltada pelas autoras é que esse processo não deve se limitar a atestar aquilo que já aconteceu, mas sim auxiliar na identificação de tendências e mercados no qual a organização atua, bem como na identificação de seus concorrentes atuais e futuros.

0 processo de IC é apresentado na literatura por meio de um ciclo. Este é apontado por Kahaner (1996) como a unidade básica do processo e é composto pelas fases de planejamento e direção, coleta, análise e disseminação. Esse mesmo ciclo é um pouco mais detalhado por Herring (1999), que o chama de ciclo de inteligência tradicional (Figura 1).
Nesse ciclo, Herring (1999) demonstra claramente as etapas do processo de IC, percebe-se que quatro delas são as principais e unindo os conceitos desse autor aos de Kahaner (1996) pode-se chegar à seguinte definição de cada uma delas:

- Planejamento e condução - etapa em que são identificadas as necessidades de informação dos usuários e também é quando se realiza o planejamento de toda a atividade, ou seja, como serão delineadas as etapas subsequentes.

- Coleta - consiste na busca, coleta e organização da informação que responderá às necessidades levantadas na fase anterior e que dará subsídio para a criação dos produtos de inteligência.

- Análise e produção - momento em que é criado o produto final que deverá responder aos questionamentos apontados no início, durante a primeira etapa. Com base nas informações coletadas na fase anterior, o analista combina seus conhecimentos com as informações coletadas para auxiliar na definição de alternativas, identificação de oportunidades e ameaças, minimização das fraquezas e fortalecimento das forças presentes na organização.

- Disseminação - é a etapa que envolve a distribuição do produto de IC que foi solicitado. Essa entrega pode ser feita em meio físico (Ex.: relatórios em papel) ou meio digital (Ex.: repositório, portal, etc.)

Gomes e Braga (2004) ainda apontam uma quinta etapa dentro do processo de IC, a avaliação. Elas propõem uma avaliação em dois aspectos: primeiro quanto ao desempenho de cada uma das fases; e segundo junto ao usuário do sistema, sua percepção quanto aos resultados práticos obtidos com o uso dos produtos gerados pelo sistema. Essas avaliações são consideradas muito importantes para o aperfeiçoamento do sistema e para a sua sobrevivência, uma vez que o processo de IC só se consolida se a inteligência resultante for utilizada na tomada de decisão.

É importante também apontar os benefícios organizacionais advindos de um eficiente sistema de IC que, de acordo com Terra, Van Rijnbach e Kato (2000, p. 3) são:

- Antecipação a movimentos da concorrência; Deteç̧ão antecipada de tecnologias emergentes e de alto impacto estrutural; - Redução de custos 


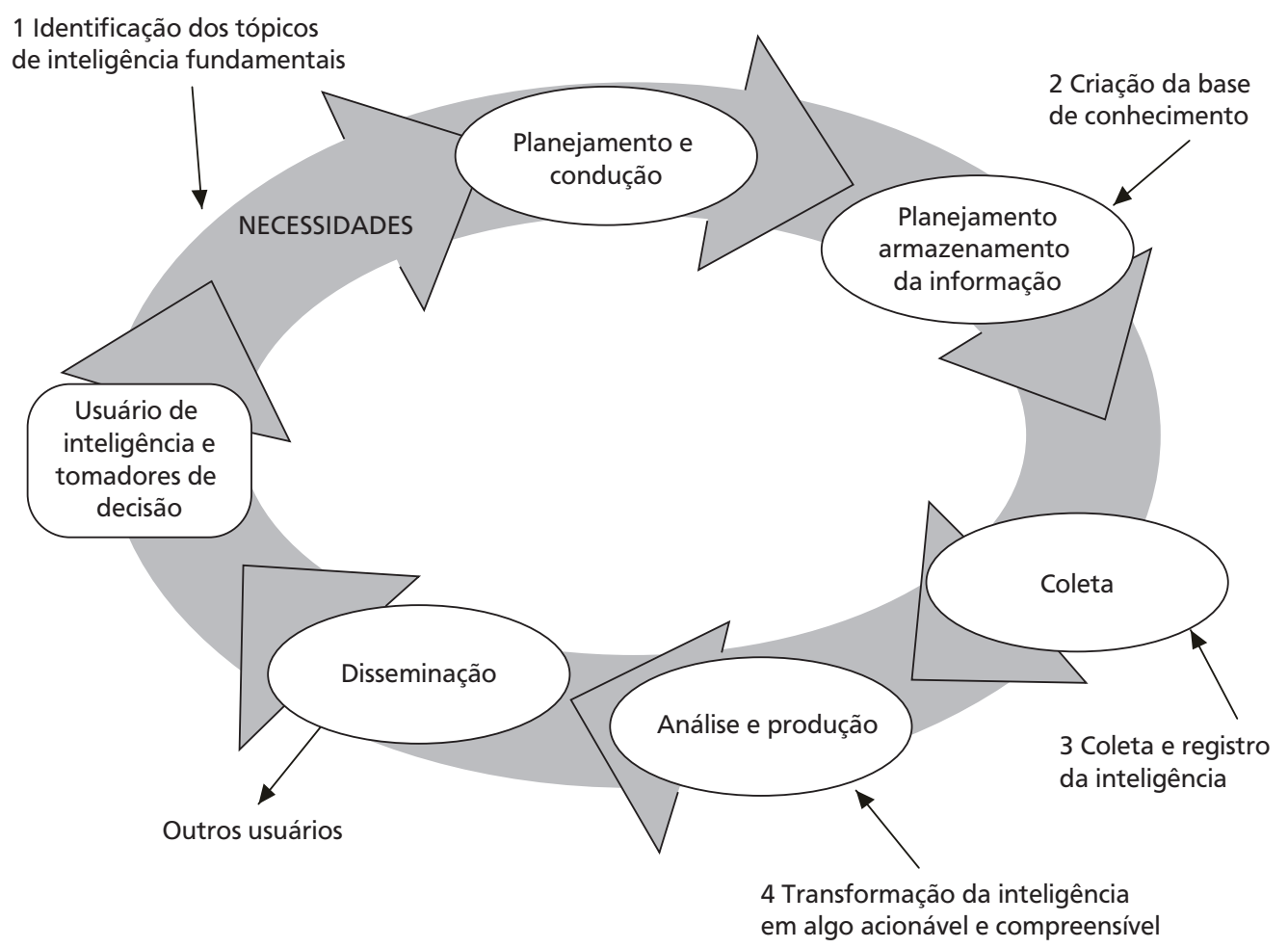

Figura 1 - Ciclo de Inteligência tradicional

Fonte: HERRING, 1999, p. 6, tradução nossa.

com o aprimoramento da performance operacional por meio de benchmarkings; - Subsídios para estratégias de fusão e aquisição; - Melhoria da eficiência das ações marketing; - Reavaliação constante das estratégias através da criação de cenários; Desenvolvimento de fornecedores mais estratégicos e competitivos em custo; - Penetração em novos mercados.

Outras vantagens podem ser proporcionadas às organizações que optam por utilizar um sistema de IC, tais como: expansão e consolidação de seus atuais mercados; identificação de oportunidades e ameaças; minimização do tempo de busca e análise de informações; apoio a trabalhos prospectivos; desenvolvimento de atitude proativa, antecipando-se às tendências; redução de duplo trabalho ou de repetição desnecessária do trabalho e custos de uma maneira geral, aumento da lucratividade; desenvolvimento de um processo contínuo de aperfeiçoamento da capacitação de profissionais; tomada de decisão baseada em conhecimento do ambiente de negócios e aprimoramento das relações da empresa com o mercado consumidor (GOMES; BRAGA, 2007).

Ao referir-se exclusivamente às MPEs, Albuquerque (2007) afirma que devido a sua agilidade na tomada e implementação de decisão, bem como alta capacidade de adaptação, a inteligência competitiva encontra um terreno fértil para o fortalecimento do ambiente competitivo e para a geração de novos negócios com base em inovação.

O case apresentado neste estudo utiliza o termo IC setorial, tendo em vista que atende às MPEs de um mesmo setor, organizadas em grupos, os Arranjos Produtivos Locais (APLs).

Hoffmann, Gregolin e Oprime (2004) explicam que o contexto econômico de alta competitividade tem provocado maior exposição das empresas brasileiras à concorrência internacional principalmente. Esse fator torna as MPEs mais vulneráveis devido aos custos de produção, escassez de recursos, etc. Portanto, houve um momento em que foi necessário rever as 
estruturas de relações entre as MPEs de um determinado setor, tendo em vista que existem muitas regiões com clara vocação econômica, impacto social e econômico, que impulsionam a união dessas empresas em prol de um objetivo comum. Os autores entendem que os APLs, além de representarem verdadeiros polos industriais, também são considerados uma ação estratégica de fortalecimento da competitividade de MPEs.

A efetividade dos sistemas de inteligência reside em uma correta disponibilização de recursos informacionais para apoiar processos decisórios, sobretudo no que diz respeito ao estabelecimento de um fluxo contínuo e sistemático de informação na forma de serviços e produtos (ARAÚJO JUNIOR; ALVARES, 2007). Mais do que isso, a inteligência pode fortalecer a cooperação entre empresas, tornando-as mais competitivas no mercado. O SIS/SEBRAE, como será apresentado, explora muito essa interação e as inúmeras oportunidades e benefícios que ela pode trazer para as MPEs frente aos mercados nacional e internacional.

\section{Metodologia da pesquisa}

A pesquisa é exploratória e a estratégia escolhida é o estudo de caso. Segundo Yin (2001), essa técnica é apropriada quando se examinam fatos ou acontecimentos e não se pode manipular comportamentos relevantes. Objetivando compreender o modelo de Inteligência Competitiva Setorial construído pelo SEBRAE/SC, a coleta de dados foi dividida em duas etapas. Na primeira etapa foi realizado um levantamento documental para se entender o modelo e as regras de negócio utilizadas na construção do Portal. Nesta etapa foram estudados e descritos, além da metodologia do projeto, os produtos de inteligência gerados a partir do sistema. Na segunda etapa foi realizada uma pesquisa bibliométrica com autores referências do tema, onde foram identificadas e analisadas as principais teorias que sustentam o tema da Inteligência Competitiva. Por fim foram comparados os achados teóricos com a metodologia empregada na construção do framework do projeto e são apresentadas as considerações finais.

\section{Sistema de inteligência setorial (SIS)}

O SIS começou a ser planejado em meados de 2007 pelo SEBRAE/SC. Em junho de 2008 o Portal SIS foi lançado para atender os APLs de vestuário, calçados femininos, móveis de madeira e apicultura, com o objetivo de oferecer a esses setores uma ferramenta para ajudar a aumentar a competitividade dos seus negócios.

Desde o início do projeto foi estabelecida interação constante entre o SEBRAE/SC e os empresários de cada setor, portanto são realizadas reuniões mensais nos APLs para reconhecer as necessidades dos empresários e também mensurar os resultados a partir das estratégias adotadas.

O Portal é uma ferramenta que funciona como interface entre o usuário, a informação e a equipe responsável pelo funcionamento do sistema. É por meio dele que os empresários fazem as solicitações de relatórios e também é onde são disseminadas as informações relativas ao ambiente de negócios dos APLs em forma de notícias, cases, artigos, relatórios, etc.

Os empresários associados aos APLs podem cadastrar-se gratuitamente e ter acesso diariamente aos seguintes recursos: agenda, biblioteca, cotações, fornecedores, legislação, notícias e relatórios. Também são disponibilizados os cases de sucesso, enquetes e a previsão do tempo. Entre todos os recursos disponíveis, os relatórios de IC são o diferencial do serviço.

Uma equipe multidisciplinar formada por profissionais especializados na coleta, tratamento, análise das informações e nas TICs são coordenados por um gestor com formação específica em IC. 0 trabalho conjunto dessa equipe proporciona a criação de produtos de IC que podem facilitar a tomada de decisão e ajudar a aumentar a competitividade das empresas (SEBRAE, 2010).

São os produtos de IC que consolidam todo o processo de IC proposto pelo SIS e eles são classificados em sete tipos:

- Alertas: são produtos simples, mas de alta velocidade de resposta. Logo que uma informação que possa gerar vantagem competitiva é detectada, é preciso fazê-la circular entre os tomadores de decisão. Geralmente são enviados por mensagem SMS ou e-mail.

- Planos de ação: apontam alternativas estratégicas de médio e longo prazo para os clientes, respondendo às demandas idenficadas no início do processo de IC. Também apresentam oportunidades e ameaças que surgem em função de eventos do ambiente organizacional.

- Sumários executivos: são documentos que abordam um tema e procuram responder de 
forma bastante direta a questões apresentadas pelo cliente na identificação das suas necessidades ou, ainda, a uma demanda específica por informação mais bruta.

- Análise de situação: uma análise de situação é um retrato "as is" de um KIT (Tópico Chave de Inteligência) ou área de monitoramento específica. Não aponta sugestões de estratégia para o cliente, já que pode servir para várias áreas da empresa.

- Relatórios analíticos: são instrumentos para tomada de decisão específica. Neles é analisada uma questão individual, relevante para o cliente. Normalmente são relatórios que avaliam uma faceta mais setorial em vez de um único concorrente ou cliente.

- Projeções estratégicas: é um documento bastante profundo e com alto grau de análise agregada. Independente do tema ou tópico que aborda, a projeção de cenários tem uma particularidade, usa tendências passadas para tentar projetar o futuro.

- Relatórios ad hoc: são os relatórios solicitados individualmente pelos empresários para atender à necessidade de informações especializadas no auxílio à tomada de decisão.

Cada empresa cadastrada pode solicitar até dois relatórios por mês, na categoria relatório ad hoc, obedecendo a um intervalo de 15 dias entre cada solicitação. 0 prazo de entrega dos relatórios é de até 15 dias úteis a contar da data de solicitação. 0 envio do relatório é feito por e-mail e após 30 dias é enviada uma ficha de avaliação do produto.

Os relatórios ad hoc têm exclusividade de uso de 0 a 120 dias, o que depende da indicação de quem solicitou o relatório. Caso o assinante não especifique por quanto tempo quer exclusividade, é concedido automaticamente o prazo de 30 dias ou mais, dependendo da definição da equipe do SIS. Após o prazo de exclusividade, o relatório é encaminhado automaticamente para os demais assinantes e também fica disponível na área específica do portal.

Entretanto, quando um relatório é criado por iniciativa do analista de um dos setores, o produto é enviado para todos os assinantes e também fica disponível no portal, sem exclusividade.

No ambiente de negócios, há a necessidade de a empresa tornar-se competitiva frente a seus concorrentes, entretanto, outras formas de obtenção de vantagem competitiva surgem e alteram as leis da competição. 0 concorrente não precisa ser necessariamente um inimigo.

Seguindo esse pensamento, as pequenas e médias empresas procuram se inserir nos novos mercados, buscando soluções criativas como, por exemplo, as iniciativas de aprendizado coletivo e processos associativos, que visam à produção de conhecimentos novos. Destaca-se uma visão mais coletiva da inovação. Percebe-se que a associação das MPEs e a formação de redes de conhecimento tornam-se fundamentais para o crescimento e o desenvolvimento econômico de qualquer sistema econômico (MEIRELLES, 2005).

No caso do SIS há uma preocupação evidente com relação ao aumento da competitividade das empresas, entretanto, como elas estão organizadas em APLs, mesmo antes do lançamento do portal já havia características marcantes de colaboração entre elas. O SIS surgiu para melhorar essa relação e possibilitar o acesso à informação estratégica que pode auxiliar na tomada de decisão e na obtenção de vantagem competitiva.

A troca e a busca por informações para a tomada de decisões em APLs foram intensificadas na década atual, pois esses modelos são os responsáveis pela competitividade de suas localidades em relação à geração e distribuição de riquezas (HOFFMANN; GREGOLIN; OPRIME, 2004).

Um mesmo produto de IC criado por iniciativa de um analista pode ser utilizado por empresas diferentes ligadas ao mesmo setor. 0 diferencial está na forma como o relatório será utilizado e se o conhecimento nele existente será colocado em prática de forma a criar inteligência para a tomada de decisão, identificação de novos mercados, ameaças e oportunidades.

Gulati (1998) afirma que toda abordagem colaborativa entre organizações privadas tem uma intenção competitiva. Seguindo essa mesma abordagem, Castilho Junior e Diniz (2005) complementam que comportamentos competitivos e colaborativos são compatíveis e complementares e auxiliam as empresas na luta por uma posição de vantagem competitiva sustentável.

Quando se trata de um relatório por demanda (relatório ad hoc), a exclusividade por tempo determinado representa certa vantagem para o solicitante. Entretanto, essa informação será compartilhada com as demais empresas posteriormente, o que não significa que, necessariamente, as outras empresas obterão os mesmos resultados, pois são pessoas 
diferentes e em situações diferentes que farão uso do mesmo relatório.

Atualmente, as TICs possibilitam níveis elevados de incentivos e alavancagem para o desenvolvimento de novas estruturas organizacionais colaborativas, gerenciando processos complexos, aumentando a troca de informações e possibilitando melhores decisões. (CASTILHO JUNIOR; DINIZ, 2005). 0 posicionamento das MPEs em APLs é um fator de fortalecimento do setor e que auxilia na busca pela eficiência e eficácia. Com a criação do SIS essas empresas passaram a contar também, de forma mais direta, com as TICs para alavancar vantagem competitiva e gerar novos conhecimentos.

\section{Considerações finais}

A IC setorial visa tornar um setor mais competitivo. Entende-se que quando a competitividade é conquistada em grupo todos ganham e auxiliam para o fortalecimento do mercado, o que demonstra o amadurecimento de todo o grupo.

Pode-se afirmar que o grande diferencial do SIS é a possibilidade de compartilhamento de informações e experiências entre empresas de um mesmo setor, que tem objetivos e mercados comuns, e ainda assim buscam obter vantagem competitiva por meio da colaboração e não necessariamente pela competição acirrada.

Para as MPE é muito importante poder contar com um sistema que monitora sistematicamente informações ambientais para apoiar proativamente a escolha da estratégia e o uso da informação para a tomada de decisão. Ainda mais importante é a possibilidade de interação com outras empresas do mesmo setor visando o fortalecimento conjunto.

O SIS possibilita aos APLs, por meio da colaboração, condições estratégicas e estruturais que podem ajudar na formação e sobrevivência das empresas a ele associadas. Portanto, o SIS pode ser entendido como ferramenta inovadora que possibilita o acesso e compartilhamento de informações e experiências para gerar inteligência entre os tomadores de decisão dentro das organizações.

Acredita-se que essas inter-relações possibilitam o progresso da MPEs rumo à busca por inovação de processos e tecnologia. Esse processo é intensificado quando as empresas reconhecem na cooperação um caminho a ser trilhado e entendem que elas podem complementar uma a outra e não somente buscar a competição.

\section{Referências}

ALBUQUERQUE, S. Inteligência competitiva setorial: aplicação \& resultados para microempresas. Revista GC Brasil, n. 5, p. 18-22, 2007. Disponível em: <http://www.scribd. com/doc/6833927/SBGC-Revista-GCBrasil-200711>. Acesso em: 10 jan. 2010.

ARAÚJO JÚNIOR, R. H. de; ALVARES, L. Gerenciamento estratégico da informação: a convergência a partir da sociedade da informação. Parcerias Estratégicas, v. 12, n. 25, p. 47-66, 2007.

CASTILHO JUNIOR, N.; DINIZ, E. H. Implementação e uso de colaboração digital entre organizações. In: ALBERTIN, A. L.; ALBERTIN, R. M. de M. (Org.). Tecnologia de informação: desafios da tecnologia de informação aplicada aos negócios. São Paulo: Atlas, 2005.

GOMES, E.; BRAGA, F. Inteligência competitiva: como transformar informação em um negócio lucrativo. 2. ed. Rio de Janeiro: Campus, 2004.

GOMES, E.; BRAGA, F. Termo de referência para atuação do sistema SEBRAE em inteligência competitiva. Brasília: SEBRAE, 2007.

GULATI, R. Alliances and networks. Strategic Management Journal, v. 19, p. 293-317, 1998.

HERRING, J. P. Key intelligence topics: a process to identify and define intelligence needs. Competitive Intelligence Review, v. 10, n. 2, p. 4-14, 1999.

HOFFMANN, W. A. M.; GREGOLIN, J. A. R.; OPRIME, P. C. A contribuição da inteligência competitiva para o desenvolvimento de arranjos produtivos locais: caso Jaú-SP. Revista Eletrônica de Biblioteconomia e Ciência da Informação, Florianópolis, 2004. Disponível em: <http:// www.periodicos.ufsc.br/index.php/eb/article/viewFile/290/5279>. Acesso em: 10 mar. 2010.

KAHANER, L. Competitive intelligence: how to gather, analyze, and use information to move your business to the top. New York: Touchstone, 1996.

MACHADO, C. dos R.; ABREU, A. F. de. Desafios da etapa de análise em processos de inteligência competitiva. In: KNOWLEDGE MANAGEMENT, 2009, Salvador. Anais... Salvador: SBGC, 2009. 
MEIRELLES, D. C. A inovação através do aprendizado coletivo em um contexto pós-moderno. In: STAREC, C.; GOMES, E. B. P.; CHAVES, J. B. L. (Org.). Gestão estratégica da informação e inteligência competitiva. São Paulo: Saraiva, 2005.

PORTER, M. E.; MILLAR, V. E. Como a informação proporciona vantagem competitiva. In: PORTER, M. E. Competição: estratégias competitivas essenciais. 10. ed. Rio de Janeiro: Campus, 1999.

SERVIÇO DE APOIO ÀS MICRO E PEQUENAS EMPRESAS SEBRAE. Sistema de inteligêcia setorial - SIS. 2010. Disponível em: <http://www.sebrae.com.br/uf/santa-catarina/produtos-e-servicos/sistema-de-inteligencia-setorial>. Acesso em: 25 mar. 2010.

TARAPANOFF, K. Referencial teórico: introdução. In: TARAPANOFF, K. (Org.). Inteligência organizacional e competitiva. Brasília: UNB, 2001.
TERRA, J. C.; VAN RIJNBACH, C.; KATO, D. Inteligência competitiva: roteiro de filme ou trabalho de formiguinha? Biblioteca Terra Forum. 2000. Disponível em: <http:// www.terraforum.com.br/biblioteca/Documents/libdoc00000223v001Inteligencia\%20Competitiva-roteiro\%20 de\%20filme.pdf>. Acesso em: 10 dez. 2009.

TYSON, K. The complete guide to competitive intelligence: gathering, analyzing, and using competitive intelligence. Chicago: Kirk Tyson International, 1998.

YIN, R. K. Estudo de caso: planejamento e método. 2. ed. Porto Alegre: Bookman, 2001.
Recebido: 15/06/2011

Received: 06/15/2011

Aprovado: 17/08/2011

Approved: 08/17/2011 